\title{
Classification of colorectal carcinoma obtained from the combination of DNA ploidy and genetic alterations serves as a significant prognostic factor
}

\author{
Akira Ichikawa - Kenjiro Kotake - Takashi Matsui • \\ Yasuo Koyama • Koichi Suda • Takashi Yao
}

Received: 24 July 2008/ Accepted: 31 December 2008

(C) Springer 2009

The editorial board of the Journal of Gastroenterology hereby retracts the article by Akira Ichikawa et al., "Classification of colorectal carcinoma obtained from the combination of DNA ploidy and genetic alterations serves as a significant prognostic factor", Journal of Gastroenterology [DOI: 10.1007/s00535-009-0035-9]. The work was published as an Online First article; however, ethical violations were confirmed after its publication.

A. Ichikawa $(\bowtie)$

Department of Laboratory Medicine,

Tochigi Cancer Center, 4-9-13, Yohnan,

Utsunomiya, Tochigi 320-0834, Japan

e-mail: akasichi@yahoo.co.jp; aichikaw@tcc.pref.tochigi.jp

A. Ichikawa $\cdot$ K. Suda $\cdot$ T. Yao

Department of Pathology, Juntendo University

School of Medicine, 2-1-1, Hongo, Bunkyo-ku,

Tokyo 113-8421, Japan

K. Suda

e-mail: Koichi.suda@tokushukai.jp; ksuda@med.juntendo.ac.jp

T. Yao

e-mail: tyao@med.juntendo.ac.jp

K. Kotake $\cdot$ T. Matsui $\cdot$ Y. Koyama

Department of Surgery, Tochigi Cancer Center, 4-9-13,

Yohnan, Utsunomiya, Tochigi 320-0834, Japan

K. Kotake

e-mail: kkotake@tcc.pref.tochigi.jp

T. Matsui

e-mail: tmatsui@tcc.pref.tochigi.jp

Y. Koyama

e-mail: ykoyama@tcc.pref.tochigi.jp

\section{K. Suda}

Department of Pathology,

Tokyo-West Tokushukai Hospital,

3-1-1, Matsubara-cho, Akishima,

Tokyo 196-0003, Japan 